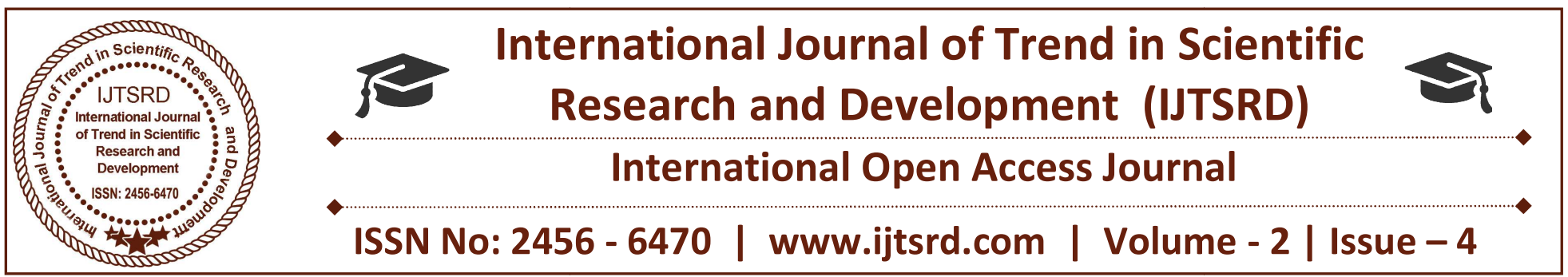

\title{
Biometric Finger Print Identification using DWT Byreal Minutiaeextraction
}

\author{
Mradula Jain ${ }^{1}$, Anshul Khurana ${ }^{2}$ \\ ${ }^{1}$ Research Scholar, ${ }^{2}$ Assistant Professor \& Guide \\ M. Tech, Shri Ram Institute of Technology, Jabalpur, Madhya Pradesh, India
}

\section{ABSTRACT}

In this work an attempt is made to enhance biometric fingerprint for identification \& recognition using Discrete Wavelet Transform (DWT). In conventional biometric systems Fast Fourier Transform has been used, use of the wavelets provides higher degree of resolution enhancement. In this work first the biometric image is taken as input from stored database, then it undergone through histogram equalization, which makes the pixel value distribution of an image to increase the perceptional image information. After that resolution enhancement is done by Discrete Wavelet Transform. Then the image is binarized, followed by segmentation is used to locate the objects \& boundaries like the lines \& curves present in an images. Then, the partitioning of the fingerprint image is done into distinguished blocks specific size. After that ROI extraction is performed. In Minutia extraction there are two steps involved, named Ridge thinning \& minutia marking. Then the post processing stage consists of False minutia removal, unify termination \& bifurcation and minutiae matching. Finally, the enhanced fingerprints are matched for performance evolution of the proposed technique.

Keywords: Minutiae extraction, Wavelet Transform, minutiae matching, fingerprint, biometrics, fingerprint enhancement.

\section{INTRODUCTION}

Fingerprint recognition or fingerprint authentication refers to the automated method of verifying a match between two human fingerprints. Fingerprints are one of many forms of biometrics used to identify an individual and verify their identity. Because of their uniqueness and consistency over time, fingerprints have been used for over a century, more recently becoming automated (i.e. a biometric) due to advancement in computing capabilities. Fingerprint identification is popular because of the inherent ease in acquisition. For a long time, researchers had come up with many approached identify and verify a fingerprint. Although the position, type of minutiae, ridges and many other parameters are used, the results are still not satisfied in recognizing fingerprint. However, all fingerprint recognition verification or identification, are eventually based on a well-defined representation of a fingerprint. The underlying principle of well-defined representation of a fingerprint and matching remains the same. The verification would be straightforward if the representation of fingerprints remains the uniqueness and kept simple.

\section{FINGER IDENTIFICATION SYSTEM}

An identification system can be defined as the one which helps in identifying the individual from many people available. It generally involves matching available biometrics feature like fingerprint with the fingerprints which are already enrolled in the database.

A fingerprint identification system constitutes of fingerprint acquiring device, image pre-processes and minutia extractor and minutia matcher.

During the fingerprint image pre-processing stage, Histogram Equalization and Fourier Transform were deployed to perform image enhancement. And then the fingerprint image is binarized using the locally adaptive threshold method. The image segmentation 
task is fulfilled by a three-step approach: block direction estimation, segmentation by direction intensity and Region of Interest extraction by Morphological operations. Most techniques used in the pre-processing stage are developed by other researchers but they were structured as a new combination in this work through various tests.

The minutia matcher chooses any two minutiae as a reference minutia pair and then matches their associated ridges first. If the ridges match well, two fingerprint images are aligned and matching is conducted for all remaining minutiae.

\subsubsection{ENHANCEMENT THROUGH WAVELET TRANSFORM}

A signal is passed through number of filters for decomposition. First it is passed through low pass filter to give approximation coefficients and this signal is also decomposed by high pass filter that gives detail coefficients. After that 2nd down sampling is performed on the filters outputs.

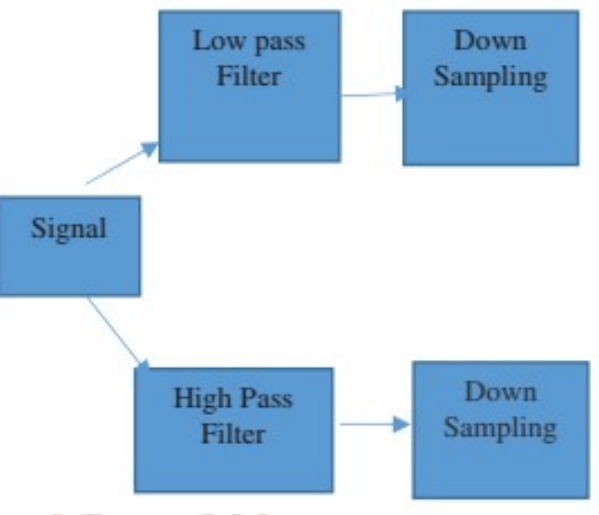

Figure 3: Wavelet Decomposition

Figure 1: Basic Fingerprint Identification System

\section{PROPOSED METHODOLOGY}

\subsection{FINGERPRINT IMAGE ENHANCEMENT}

Fingerprint image enhancement is to prepare the image to be better to ease further operations. Since the fingerprint images acquired from camera or other sensors are not guaranteed with great quality, thus image enhancement need to be carried out.

\subsubsection{ENHANCED BY USING HISTOGRAMEQUALIZATION}

The fingerprint images were first enhanced by using Histogram Equalization. Histogram is a process that attempts to spread out the gray levels in an image so that they are evenly distributed across their range. It basically reassigns the brightness value of each pixel based on the image histogram. Histogram is a technique to produce more visually pleasing result across a wider range of images to produce as flat as possible histogram of the image.

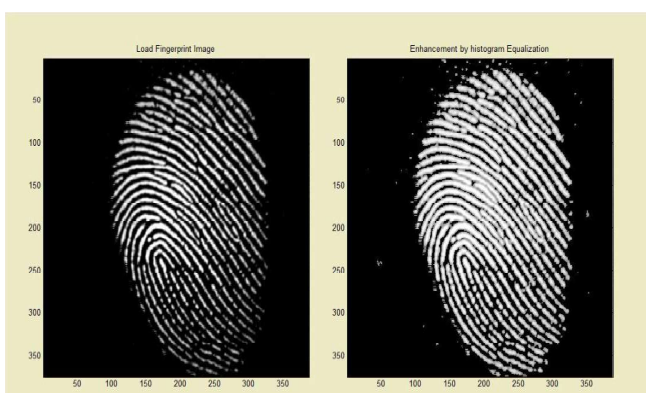

Figure 2: Histogram Enhancement (left: original image, right: enhanced image)
Wavelet transforms are multi-resolution image decomposition tool that offer a diversity of channels expressive the image feature by dissimilar frequency sub bands at multi-scale. It is a well-known technique in analysing signals. When putrefaction is executed, the approximation and detail component can be separated 2-D Discrete Wavelet Transformation (DWT) translates the image from the spatial domain to frequency domain. The image is divided by vertical and horizontal lines and represents the first-order of DWT, and the image can be separated with four parts those are LL1, LH1, HL1 and HH1.

To apply wavelet transform in images, the extension to $2 \mathrm{D}$ has to be made. For 2D data, the transform can be categorized into separable and non-separable transform. Most discrete wavelet transform in 2D data is performed using the separable wavelet transform. For the separable transform, the two dimensional wavelets are defined as tensor products of 1D wavelets. This results in one scaling function and three different mother wavelets:

$\phi[i, j]=\phi[i] \phi[j]$

$\Psi_{1}[i, j]=\phi[i] \Psi[j]$

$\Psi_{2}[i, j]=\Psi[i] \phi[j]$

$\Psi_{3}[i, j]=\Psi[i] \Psi[j]$

This type of product allows one dimensional filtering of rows, followed by one dimensional filtering of columns, instead of two dimensional filtering. This results in four different filtered images (Figure 4.5); one of which represent the approximation, namely the 
low-low (LL) channel, while the other three represent the details, namely the low-high (LH) channel, highlow (HL) channel and high-high (HH) channel. LL is a smoothed version of the original image, while the detail images $\mathrm{LH}, \mathrm{HL}$ and $\mathrm{HH}$ contain respectively the details of the vertical, horizontal and diagonal directions, thus retaining specific orientation information. After this, a sub sampling in both directions can be performed. As in the one dimensional case, by iterating the procedure on successive low pass images LL, sub images LL on different levels are generated. This results in a pyramid with detail images for different scales and orientations, and is called two dimensional standard or pyramidal wavelet decomposition.

Decomposition
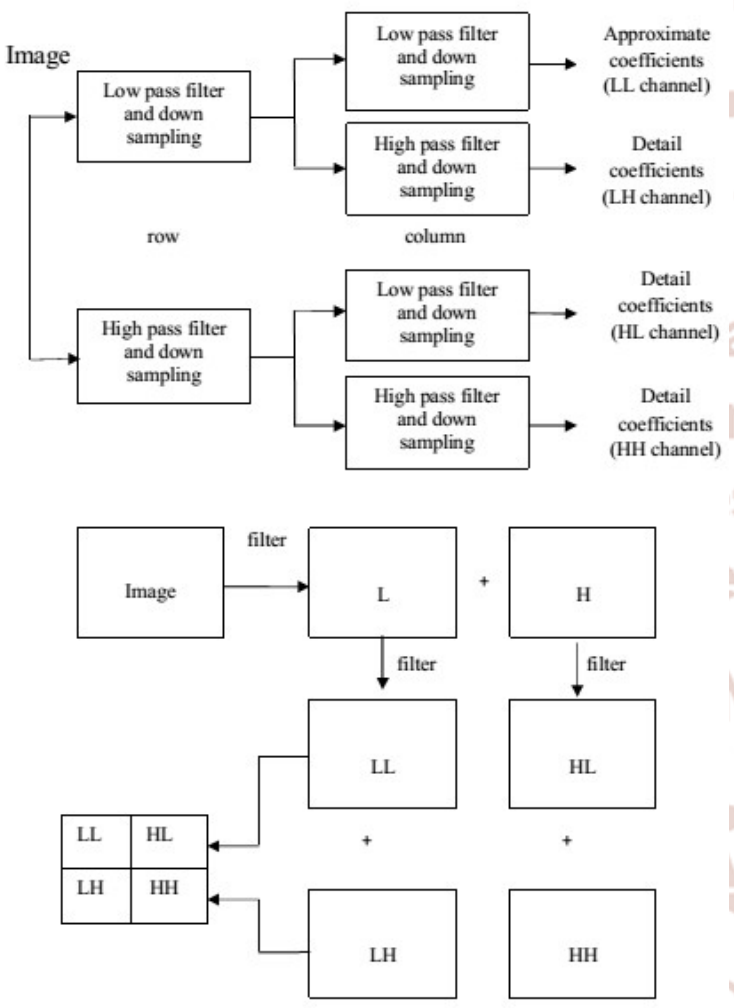

Figure 4: One level wavelet decomposition of two dimensional data

In this enhancement, the image is divided into small processing blocks of 32 by 32 pixels and then we perform the Wavelet Transform on each block according older concept of FFT as:

$$
\begin{aligned}
F(u, v)=\sum_{x=0}^{M-1} & \sum_{y=0}^{N-1} f(x, y) \\
& \times \exp \left\{-j 2 \pi \times\left(\frac{u x}{M}+\frac{v y}{N}\right)\right\}
\end{aligned}
$$

for $u=0,1,2, \ldots, 31$, and

for $v=0,1,2, \ldots, 31$.
In order to enhance each block by its dominant frequencies, each block after DWT will be multiplied with its magnitude a set of times. Where magnitude can be given as:

$\operatorname{abs}(F(u, v))=|F(u, v)|$

and the enhanced block will be based on:

$$
g(x, y)=F^{-1}\left\{F(u, v) \times|F(u, v)|^{k}\right\}
$$

where $F^{-1}\{F(u, v)\}$ is given by

$$
\begin{aligned}
F(x, y)=\frac{1}{M N} \sum_{x=0}^{M-1} \sum_{y=0}^{N-1} F(u, v) \\
\quad \times \exp \left\{j 2 \pi \times\left(\frac{u x}{M}+\frac{v y}{N}\right)\right\}
\end{aligned}
$$

for $x=0,1,2, \ldots, 31$, and

for $y=0,1,2, \ldots, 31$.

The $\mathrm{k}$ in the formulae is a constant which is determined experimentally, here we will choose the $\mathrm{k}$ value $=0.45$ by some experiments over fingerprints. Suppose, if we have a higher ' $\mathrm{k}$ ' then the appearance of the ridges will be improved and it will fill up the small holes in ridges but, if have a very higher ' $k$ ', then it can result into false joining of ridges. Hence, termination minutiae might become bifurcation minutiae.

Figure 5 represents the image after DWT enhancement where figure 6 is the image after histogram equalization

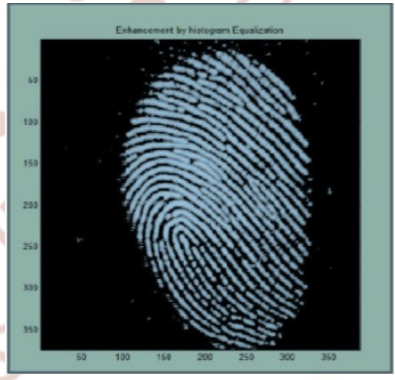

Figure 5: Histogram equalized image

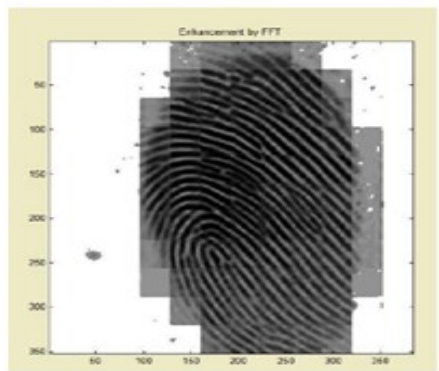

Figure 6: Image after DWT operation

\subsubsection{FINGERPRINT IMAGE BINARIZATION}

Fingerprint image binarization is done to transform a 8-bit gray image to a 1-bit binarized image where 0value holds for ridges and 1-value for furrows. And 
after the binarization operation ridges are highlighted with black color and furrows are highlighted with white color.

Here, we will use a locally adaptive binarization method called as 'adaptive thresholding' to binarize the fingerprint image. In this method we transform the gray level to 0 if it is below threshold value and to 1 if it is above threshold value. The threshold value is the mean taken from the gray level of the current block $(16 * 16)$ to which the pixel belongs [Figure7].
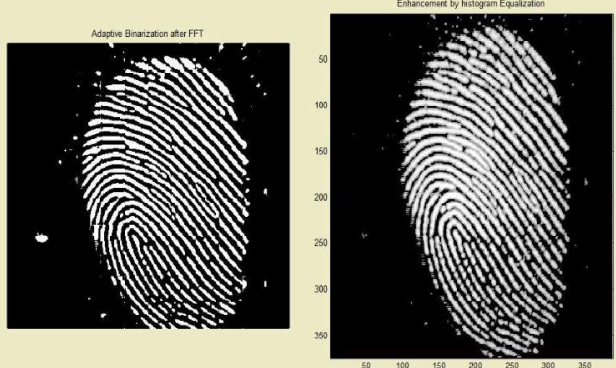

in fingerprint recognition thinning is done to thin the ridges so that each is one pixel thick. In each scan of the fingerprint image, the algorithm removes the redundant pixels in small image window $(3 \times 3)$.In our algorithm, for thinning purposes we had invoked an inbuilt morphological operation in MATLAB.
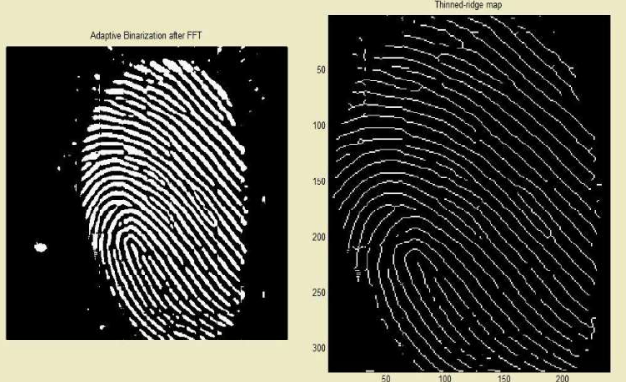

Figure 9: (a) Binarized Image (b) Thinned Ridge Image

\subsection{MINUTIA MATCH}

The minutia details of two fingerprints are obtained using the above procedures and they are matched using the minutia match algorithm. Alignment based match algorithm is used in our project. It comprises of two stages:

1. Alignment Stage.

2. Match Stage.

As for our aim only region of interest is the useful part which needs to be recognized for each and every fingerprint image. Here, the image area without effective furrows and ridges will be first discarded from the image since it has only background information. Then we will sketch out the bound of the remaining effective area since bound region minutiae produces confusion with the spurious minutiae that are generated out of the sensor.

To get the ROI we use a two-step method. The first step constitutes 'block direction estimation' and 'direction variety check', whereas the second step is done using some morphological operations.

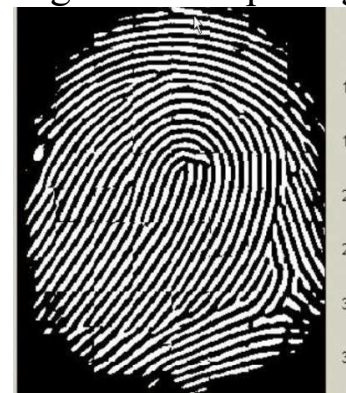

Figure 8: Direction Flow Estimate Binarized Image (Left), Direction Map (Right)

\section{MINUTIAE EXTRACTION}

\subsection{FINGERPRINT RIDGE THINNING}

Thinning is the process of reducing binary objects or shapes to strokes whose width is one pixel wide. Here
An iterative ridge alignment algorithm is first used to align one set of minutia with respect to another and then an elastic match algorithm is carried out to count the number of matching minutia pairs.

\subsection{PROPOSED METHODOLOGY}

In this an attempt is made to enhance biometric fingerprint identification \& recognition using wavelet transform. In conventional biometric systems Fast Fourier Transform has been used, use of the wavelets provides higher degree of resolution enhancement. In this work first the biometric image is taken as input from stored database, then it undergone through histogram equalization process, which converts the distribution of pixel value of an image to increase the perceptional information of the image. After that resolution enhancement is done by Discrete Wavelet Transform. Then the image is binarized, followed by segmentation which is used for locating the objects, boundaries, lines and curves of an image. Then, the partitioning of the fingerprint image is done into distinguished blocks specific size. After that ROI extraction is performed. In Minutia extraction there are two steps involved, named Ridge thinning \& minutia marking. Then the post processing stage consists of false minutia removal, unify termination \& 
bifurcation and minutiae matching. Finally, the enhanced fingerprints are matched for performance evolution of the proposed technique. The proposed procedure follows the below steps;

1. Fingerprint Image Enhancement by

H. E. \& D. W. T.

2. Fingerprint Image Binarization.

3. Fingerprint Image Segmentation.

4. Block Direction Estimation.

5. ROI Extraction.

6. Minutia Extraction.

7. Post Processing.

8. Performance Evolution.

The flow chart of the proposed system is shown in figure 9 .

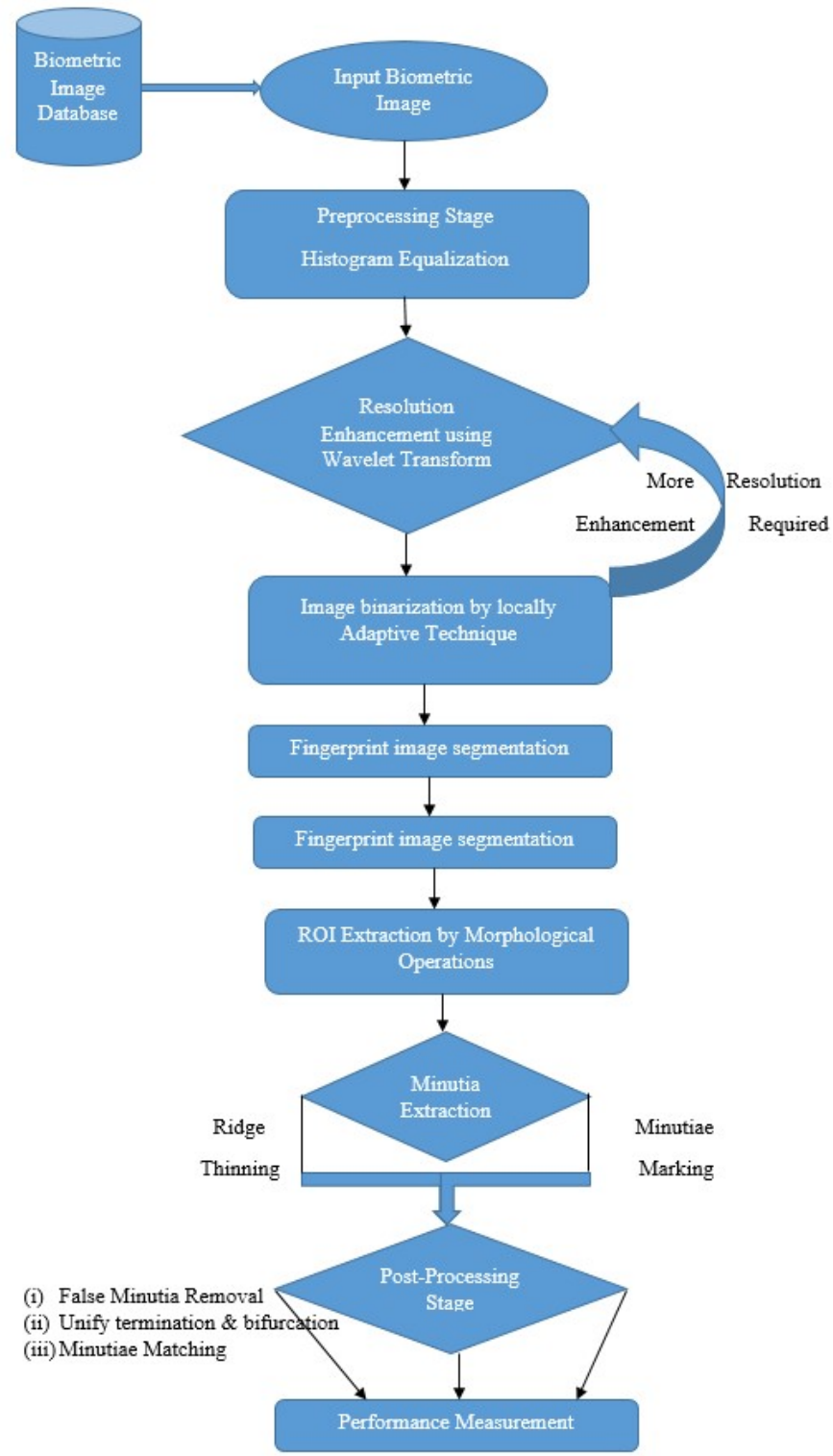

Figure 9: Proposed Biometric Fingerprint Identification Algorithm

\section{SIMULATION RESULTS}

This work used fingerprint database which includes fingerprint of 16 persons and each of them with 8 image fingerprint and matching score was obtained in order to compare same image and finally the recognition performance analyzed.

\subsection{PERFORMANCE PARAMETERS}

To determine the performance of a fingerprint recognition system False Rejection Rate (FRR) is most commonly used as performance evaluation index. Sometimes the biometric security system may incorrectly reject an access attempt by an authorized user. To measure these types of incidents FAR is basically used. A system's FRR basically states the ratio between the number of false rejections and the number of identification attempts.

Mathematically FRR may be expressed in the following manner:

$$
\text { (\%) } F R R=(F R / N) * 100
$$

$F R=$ number of incidents of false rejections

$N=$ number of samples

The FRR depends upon the quality of the image whether the quality is good or bad.

The recognition of fingerprint sample will be done \& processed in the following manner using MATLAB environment.

Step1. Loading the fingerprint database .

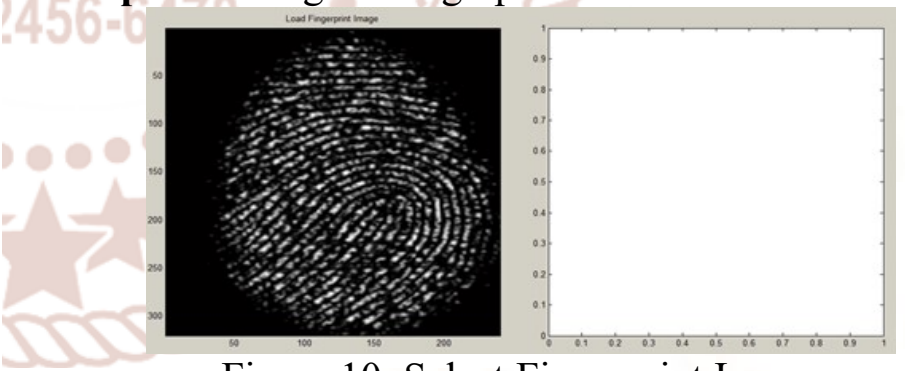

Step2.

Figure 10: Select Fingerprint Image

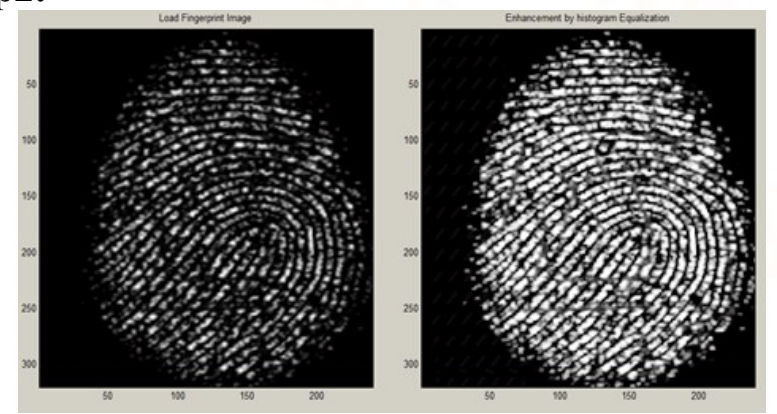

Figure 11: Pre-processing of selected image 
International Journal of Trend in Scientific Research and Development (IJTSRD) ISSN: 2456-6470

Step 3.
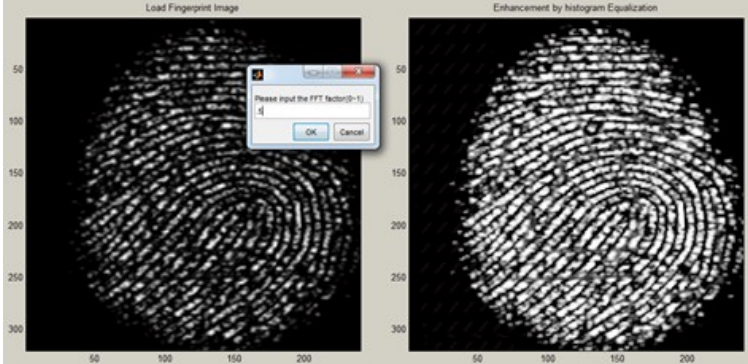

Figure 12: Perform Wavelet Decomposition Step 4.
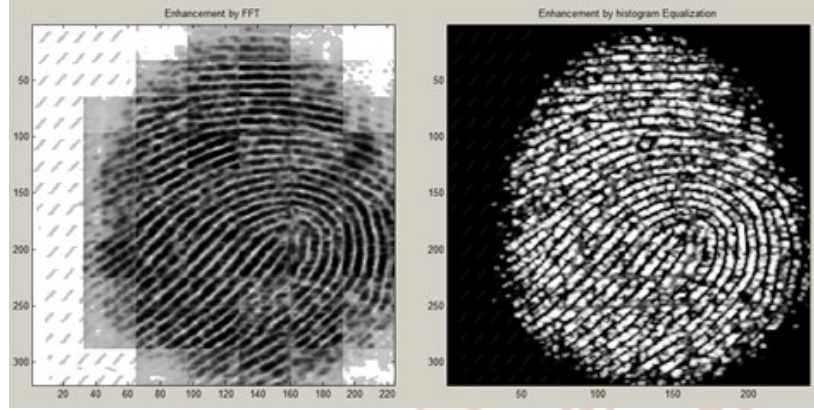

Figure 13: Enhancement by DWT

Step 5.
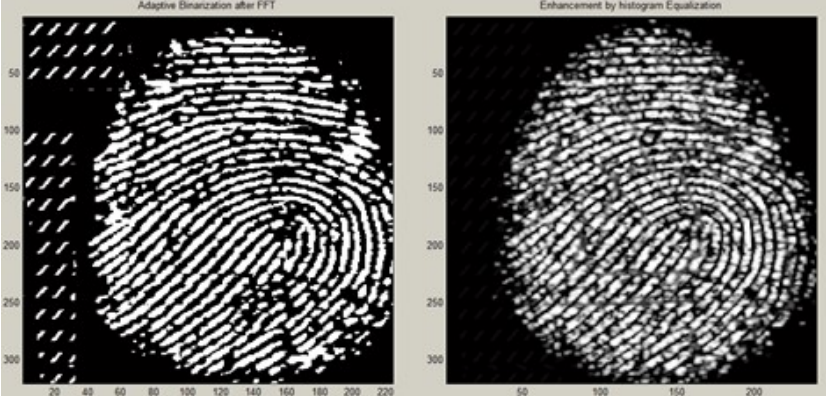

Figure 14: Enhancement by Histogram Equalization Step 6.

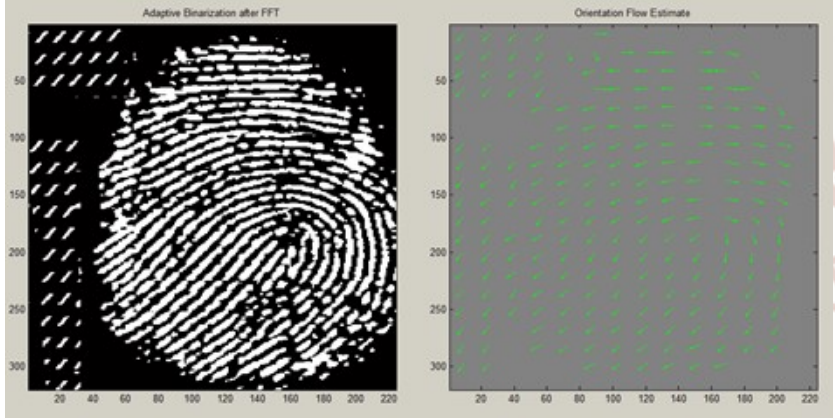

Figure 15: Orientation Flow Estimate

Step 7.

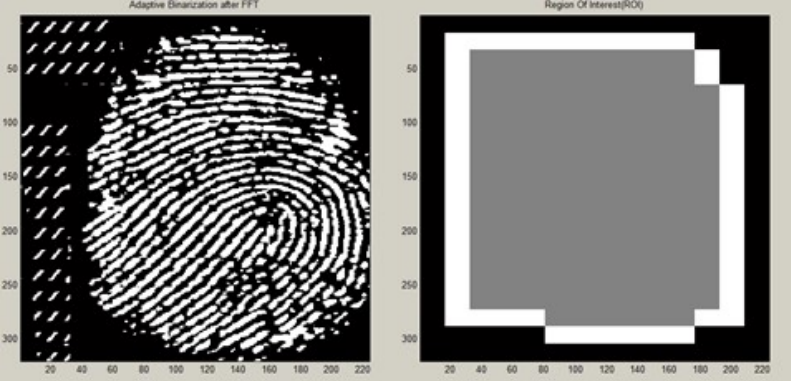

Figure 16: ROI Selection
Step 8.
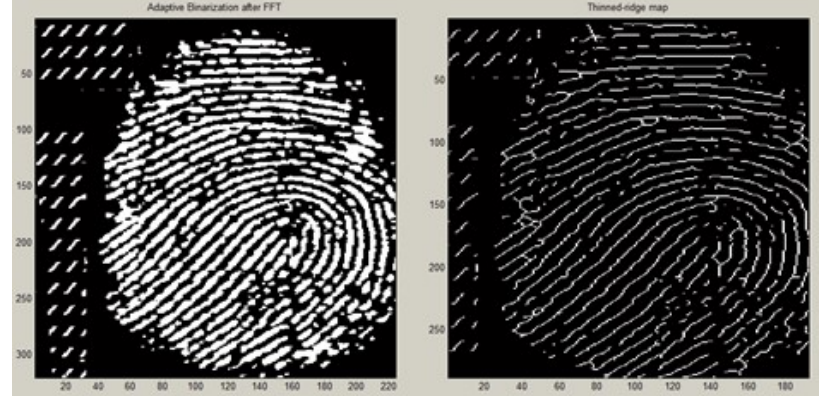

Figure 17: Thin Edge Map

\section{Step 9.}

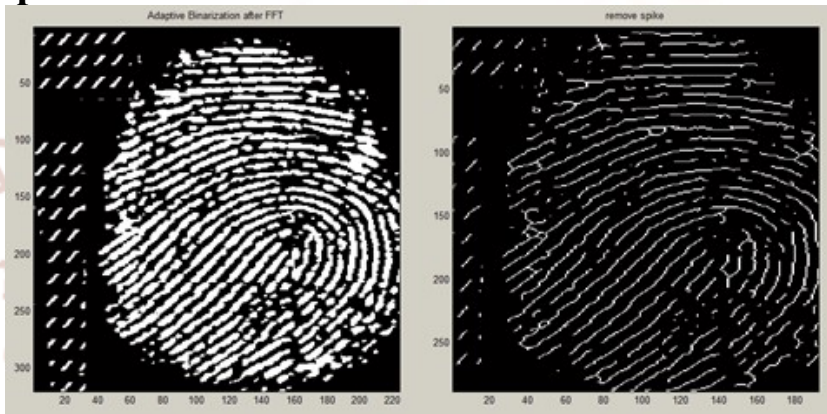

Figure 18: Spike Removal

Step10.
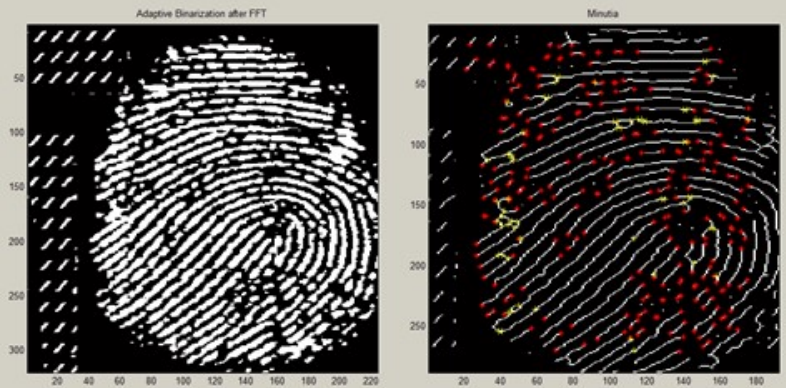

Figure 19: Minutia Extraction

Step 11.
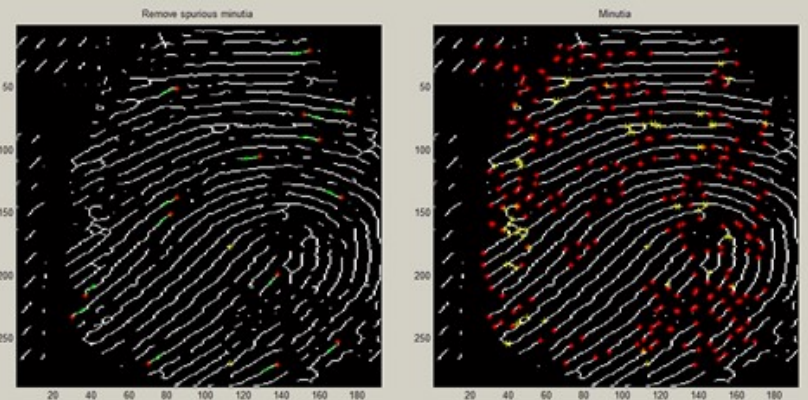

Figure 20: Identification of Real Minutia after Extraction

We start from first person the database of fingerprint per person is 8 and recognition match $7 / 8$ and percentage of matching is $87.5 \%$ but false rejection ratio (FRR) is $1 / 8$ and percentage of FRR is $12.5 \%$. Second person have the same database of fingerprint therefore same percentage matching of FRR result but the third person have database of fingerprint per person is 8 and recognition match $8 / 8$ and FRR is $0 / 8$ 
thus the percentage of matching $100 \%$ and percentage of FRR is $0.0 \%$. The eleventh person has the database of fingerprint 8 and recognition match $6 / 8$ and FRR $2 / 8$ therefore percentage of matching $75.0 \%$ and percentage of FRR 25.0\%. Overall 16 person weighted average percentage of matching is $89.062 \%$ and FRR $10.937 \%$. In the reference [3] the false rejection rate (FRR) is $23.7 \%$ due to major variation of finger print ridges depth. . Hence, an overall improvement is $12.76 \%$ is achieved in this work. Table 1 showing the FRR and recognition rate of fingerprint matching.Results comparison is shown in table 2.

\begin{tabular}{|c|c|c|c|c|c|}
\hline S. No. & $\begin{array}{c}\text { Database Of Fingerprint } \\
\text { Per Person }\end{array}$ & Recognition Match & FRR & $\%$ of Matching & $\%$ of $F R R$ \\
\hline 1 & 8 & $7 / 8$ & $1 / 8$ & $87.5 \%$ & $12.5 \%$ \\
\hline 2 & 8 & $7 / 8$ & $1 / 8$ & $87.5 \%$ & $12.5 \%$ \\
\hline 3 & 8 & $8 / 8$ & $0 / 8$ & $100.0 \%$ & $0.0 \%$ \\
\hline 4 & 8 & $7 / 8$ & $1 / 8$ & $87.5 \%$ & $12.5 \%$ \\
\hline 5 & 8 & $7 / 8$ & $1 / 8$ & $87.5 \%$ & $12.5 \%$ \\
\hline 6 & 8 & $7 / 8$ & $1 / 8$ & $87.5 \%$ & $12.5 \%$ \\
\hline 7 & 8 & $8 / 8$ & $0 / 8$ & $100.0 \%$ & $0.0 \%$ \\
\hline 8 & 8 & $7 / 8$ & $1 / 8$ & $87.5 \%$ & $12.5 \%$ \\
\hline 9 & 8 & $7 / 8$ & $1 / 8$ & $87.5 \%$ & $12.5 \%$ \\
\hline 10 & 8 & $8 / 8$ & $0 / 8$ & $100.0 \%$ & $0.0 \%$ \\
\hline 11 & 8 & $6 / 8$ & $2 / 8$ & $75.0 \%$ & $25.0 \%$ \\
\hline 12 & 8 & $8 / 8$ & $0 / 8$ & $100.0 \%$ & $0.0 \%$ \\
\hline 13 & 8 & $6 / 8$ & $2 / 8$ & $75.0 \%$ & $25.0 \%$ \\
\hline 14 & 8 & $7 / 8$ & $1 / 8$ & $87.5 \%$ & $12.5 \%$ \\
\hline 15 & 8 & $7 / 8$ & $1 / 8$ & $87.5 \%$ & $12.5 \%$ \\
\hline 16 & 8 & $7 / 8$ & $1 / 8$ & $87.5 \%$ & $12.5 \%$ \\
\hline & \multicolumn{3}{|c|}{ Weighted Average Percentage } & $89.062 \%$ & $10.937 \%$ \\
\hline
\end{tabular}

Table 1: Simulation Results Summary

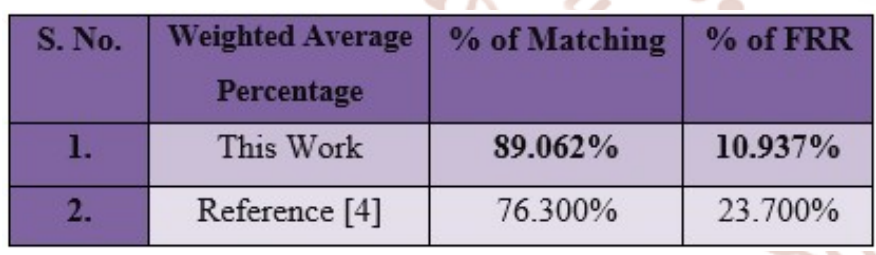

Table 2: Simulation Results Comparison

\section{Simulation Results Comparison}

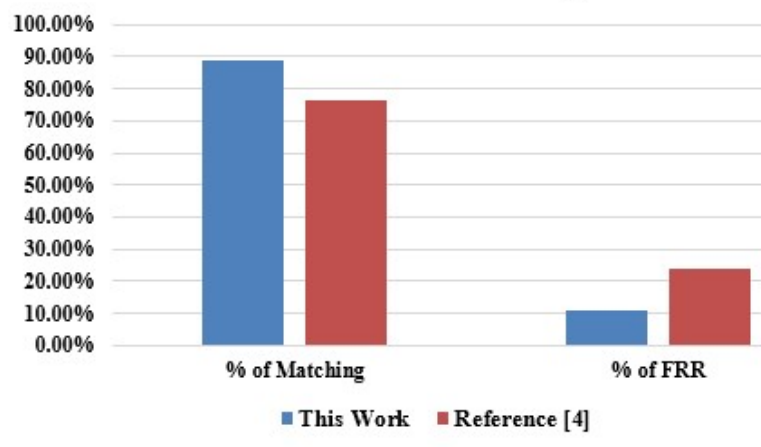

Figure 21: Graph of Simulation Results Comparison

\section{CONCLUSION}

This work focuses on image enhancement that include noise reduction, smoothing, contrast stretching, Fourier transform and edge enhancement and from that presented a practical performance for minutiae extraction and recognition of the fingerprint images. The matching score of the minutiae was observed to increase at a lower enhancement threshold and by limiting the minimum number of minutiae extracted prior to recognition. These results validate that the collection of image enhancement technique can further improve the recognition rate and matching score by using minutiae extraction. It is believed that further research can be made in producing a better slight image rotation and filtration of unsuitable images that lacking criteria for fingerprint detection.

\section{REFERENCES}

1. Muzhir Shaban et-al, "Effective Fingerprint Recognition Approach Based on Double Fingerprint Thumb", International Conference on Current Research in Computer Science and Information Technology (ICCIT), Iraq, 2017.

2. C. Kauba et-al, "Fingerprint recognition under the influence of image sensor ageing", IEEE IET journal International Workshop on Biometrics and Forensics (IWBF), 2017.

3. U. M. Tomar et-al, "Fingerprint Recognition by Hybrid Optimization Based On Minutaies Distance and Pattern Matching”, International conference on Signal Processing, Communication, Power and Embedded System (SCOPES)-2016.

4. Sehasnainjot Singh et-al, "Finger Print Fusion Using Daubechies (Db1) Wavelet Transformation \& Quality Measures", 2nd IEEE International Conference on Computing for Sustainable Global Development (INDIACom), Pp. 822-826, 2015.

5. R. Priya et-al, "A Novel algorithm for Secure Internet Banking with finger print recognition", IEEE International Conference on Embedded Systems (ICES), Pp.104-109, Coimbatore, 2014.

6. Sayak Bhowal et-al, "A Case Study of Illumination Robust Face \& Finger Print Merging Identification Technique using Cross Correlation”, IEEE International Conference on Emerging Trends in Communication, Control, Signal Processing \& Computing Applications (C2SPCA), 2013.

7. Dr. V. Vijayalakshmi et-al, "Finger \& Palm print based Multibiometric Authentication System with 
International Journal of Trend in Scientific Research and Development (IJTSRD) ISSN: 2456-6470

GUI Interface”, IEEE International conference on Communication \& Signal Processing, Pp. 738$742,2013$.

8. Smital D. Patil \& Shailaja A. Patil, "Fingerprint Recognition Using Minutia Matching", in Proceedings of "Conference on Advances in Communication \& Computing (NCACC'12)", 2012.

9. M. N. Shah Zainudin, Radi et-al ., "Face Recognition using Principle Component Analysis (PCA) \& Linear Discriminant Analysis (LDA)", International Journal of Electrical \& Computer Sciences, Vol-12, No-05, 2012.

10. Vikram Singh \& Kalpna Kashyap, "A survey paper on "hybrid system for finger print identification", International Journal of Emerging Trends \& Technology in Computer Science (IJETTCS), Volume 1, Issue 4, 2012.

11. Vaidehi. V, Naresh Babu N T, Ponsamuel Mervin. A, Praveen Kumar. S, Velmurugan. S, Balamurali, Girish Chandra, "Fingerprint Identification Using Cross Correlation of Field Orientation," Second International Conference on Advanced Computing (ICoAC), Pp. 66- 69, 2010.

12. Ashwini R. Patil, Mukesh A. Zaveri, "Novel Approach for Fingerprint Matching using Minutiae", in Fourth Asia IEEE International Conference on Mathematical/Analytical Modeling and Computer Simulation, 2010.

13. J.A. Carrasco-Ochoa et al. (Eds.): "Fingerprint verification with Non-linear Composite Correlation Filters," MCPR 2010, LNCS 6256, pp. 90-97, Springer-Verlag Berlin Heidelberg, 2010.

14. S. L. de O. Gonzaga, "A Method for Fingerprint Image Identification Based on Gabor Filter and Power Spectrum", 2010.

15. R. N. Rodrigues, L.L. Ling, and V. Govindarju, ": Robustness of Multimodal Biometric Fusion methods against Spoof Attacks," Journal of Visual Languages Communication, vol. 20, no. 3, pp. 169-179, 2009.

16. A. Nagar and A. K. Jain, "On the Security of Noninvertible Finger Print Template Transforms," Proceedings of IEEE Workshop on Information Forensic sand Security, London, U.K, 2009.

17. Haiyun $\mathrm{Xu}$, Raymond N. J. Veldhuis, Tom A.M. Kevenaar and Ton A.H.M. Akkermans, "A Fast
Minutiae-Based Fingerprint Recognition System" in IEEE Systems Journal, Vol.3, No.4, Pp. 418427, 2009.

18. Ravi. J, K. B. Raja, Venugopal K. R, "Fingerprint Recognition Using Minutia Score Matching", International Journal of Engineering Science and Technology Vol.1 (2), Pp. 35-42, 2009.

19. D. Maltoni, D. Maio, A.K. Jain, S. Prabhakar, "Handbook of Fingerprint Recognition (Second Edition)," Springer, London, 2009.

20. A. Jain, K. Nandakumar, and A. Nagar, "Biometric Template Securtiy EURASIP Journal on Advance Signal Processing vol.4, 2008.

21. H. Xu, R. Veldhuis, T. Kevenaar, A. Akkermans, and A.Bazen," Spectral Minutiae A Fixed Length Representation of a Minutiae Set Proceedings of Biometrics, Pp. 1-6, June 2008.

22. K. Nallaperumall, A. L. Fred and S. Padmapriya, "A Novel for Fingerprint Feature Extraction Using Fixed Size Templates", IEEE 2005 Conference, Pp. 371-374, 2005.

23. P. Komarinski, P. T. Higgins, and K. M. Higgins, K. Fox Lisa , "Automated Fingerprint Identification Systems (AFIS)", Elsevier Academic Press, pp. 1-118, 2005.

24. S M Mohsen, S M Zamshed Farhan and M MA Hashem, "Automated Fingerprint Recognition: Using Minutiae Matching Technique for the Large "Fingerprint Database", 3rd IEEE International Conference on Electrical \& Computer Engineering (ICECE), Pp. 28-30, 2004.

25. D. Maltoni, D. Maio, and A. Jain, S. Prabhakar, "4.3: Minutiae-based Methods"e (extract) from Handbook of Fingerprint Recognition", Springer, New York, Pp. 141-144, 2003.

26. Salil Prabhakar, Anil K Jain and Sharath Pankanti, "Learning fingerprint minutiae location and type", Pattern recognition, Pp. 1847-1857, 2003.

27. Lin Hong, Student Member, IEEE, Yifei Wan, and Anil Jain, "Fingerprint Image Enhancement: Algorithm and Performance Evaluation" IEEE Transactions on Pattern Analysis And Machine Intelligence, Vol. 20, Pp. 777-787, 1998.

28. D. Maio, and D. Maltoni, "Direct gray-scale minutiae detection in fingerprints", IEEE Transactions Pattern Analysis and Machine Intelligence, vol. 19(1), Pp. 27-40, 1997. 